\title{
PPARס increases expression of the human apolipoprotein A-II gene in human liver cells
}

\author{
PETRA THULIN $^{1}$, BJÖRN GLINGHAMMAR ${ }^{2}$, JOSEFIN SKOGSBERG $^{1}$, \\ KERSTIN LUNDELL ${ }^{1}$ and EWA EHRENBORG ${ }^{1}$ \\ ${ }^{1}$ Atherosclerosis Research Unit, Department of Medicine, Center for Molecular Medicine, Karolinska Institutet, \\ Karolinska University Hospital, Solna, SE-171 76 Stockholm; ${ }^{2}$ AstraZeneca, Safety Assessment, \\ Molecular Toxicology, S-151 85 Södertälje, Sweden
}

Received January 17, 2008; Accepted February 25, 2008

\begin{abstract}
The peroxisome proliferator-activated receptor $\delta$ (PPAR $\delta$ ) is a transcription factor that regulates genes of importance in lipid and glucose metabolism. ApoA-II is one of the major proteins of the HDL-particle. The aim of this study was to investigate the regulation of apoA-II gene expression by PPAR $\delta$. Treatment of HepG2 cells with the PPARס specific agonist GW501516 increased apoA-II mRNA expression. Likewise, reporter gene assays using a construct containing $2.7 \mathrm{~kb}$ of the proximal apoA-II promoter showed increased activity after treatment with GW501516, both in HepG2 and in HuH-7 cells. Mutation of two putative PPAR response elements (PPREs) in this region showed that the PPRE at position -737/-717 is the functional site. Binding of PPAR $\delta$ to this site was confirmed by chromatin immunoprecipitation and gel retardation analyses. In conclusion, PPAR $\delta$ increases the expression of the human apoA-II gene in liver cells via a PPRE in the proximal promoter.
\end{abstract}

\section{Introduction}

High-density lipoproteins (HDL) are inversely correlated with the incidence of coronary artery disease (CAD) in humans and have postulated roles in the atherosclerotic process such as reverse cholesterol transport, anti-inflammation and anti-oxidation (1). The main apolipoproteins of HDLs are apolipoprotein A-I (apoA-I) and A-II (apoA-II). The antiatherogenicity of apoA-I is well supported in experimental and

Correspondence to: Dr Petra Thulin, Atherosclerosis Research Unit, Department of Medicine, Center for Molecular Medicine, Karolinska Institutet, Karolinska University Hospital, Solna, SE-171 76 Stockholm, Sweden

E-mail: petra.thulin@ki.se

Key words: peroxisome proliferator-activated receptor delta, apolipoprotein A-II, gene regulation, transcription, PPAR response element, GW501516, hepatoma cells clinical studies while the role of apoA-II in the atherosclerotic process, is less clear (2). However, there is recent data showing that apoA-II is associated with a decreased risk of cardiovascular disease (3).

Fibrates increase plasma HDL cholesterol levels and have been shown to increase the levels of both apoA-I and apoA-II in plasma, thus supporting a positive correlation between apoA-II expression and plasma HDL cholesterol. Fibrates are activators of peroxisome proliferator-activated receptor $\alpha$ (PPAR $\alpha$ ), a member of the PPAR family of ligand activated transcription factors. PPARs regulate lipid and glucose homeostasis and their natural ligands are fatty acids and fatty acid metabolites (4). PPARs regulate transcription by forming heterodimers with the retinoid $\mathrm{X}$ receptor (RXR) and binding to PPAR response elements (PPREs) in the promoters of their target genes. The PPRE consists of a direct repeat (DR) of the consensus sequence AGGTCA separated by one nucleotide, a DR1 motif. In addition, seven nucleotides in the 5'-flank of DR1 have been described to be of importance for binding to the PPRE (5). Studies regarding the effect of PPAR $\alpha$ activation using approximately $1 \mathrm{~kb}$ of the promoter of the ApoA-II gene, resulted in the identification of a functional DR1 in a regulatory region, designated as the J-element, positioned 716-734 bp upstream of the transcriptional start site $(6,7)$.

PPARס is another member of the PPAR family, which has encountered a lot of attention in recent years partly due to its beneficial effects on glucose and lipoprotein metabolism in primates. Treatment of obese rhesus monkeys with a selective PPARS agonist, GW501516, increased plasma HDL cholesterol levels by $40 \%$ and both apoA-I and apoA-II levels were elevated (8). Further, subjects obtaining GW501516 in a phase I clinical trial showed increased HDL cholesterol levels compared to placebo (9). Thus, GW501516 is a candidate drug for treatment of metabolic disorders (10).

We hypothesize that activation of PPAR increases apoA-II expression via a direct transcriptional mechanism, which might contribute to the HDL increase in plasma cholesterol levels seen in primates treated with GW501516. In this study, we aimed to identify and characterize the functional PPREs located within the proximal promoter of the human apoA-II gene. 


\section{Materials and methods}

Chemicals. GW501516 was synthesized by Synthelec AB, Sweden as described (11). Fenofibric acid (FF) was a gift from professor Per Eriksson (Karolinska Institutet, Sweden).

Cell culture. The human hepatoma cells, HuH-7, were kindly provided by Dr John McLauchlan (University of Glasgow, UK) and the human liver cell line, HepG2, was purchased from ATCC (Rockville, MD, USA). The cells were cultured in DMEM (1 g/ml glucose, Invitrogen, USA), containing $10 \% \mathrm{FBS}$, penicillin $(100 \mathrm{U} / \mathrm{ml})$ and streptomycin $(100 \mu \mathrm{g} / \mathrm{ml})$ at $37^{\circ} \mathrm{C}$ in $5 \% \mathrm{CO}_{2}$. For Taqman assays the cell medium contained $10 \%$ lipid deficient FBS, which was depleted of lipids and hormones by treatment with fumed silica (s5130, Sigma-Aldrich, USA), AG-1-X8 resin (Bio-Rad, USA) and activated dextran-coated charcoal (C6197, Sigma-Aldrich).

RNA isolation, cDNA synthesis and real-time PCR. Cells were seeded out in 6-well plates in $10 \%$ lipid deficient DMEM at a density of $1 \times 10^{6}$ cells/well, incubated for $24 \mathrm{~h}$ at $37^{\circ} \mathrm{C}$ followed by treatment with GW501516, FF or vehicle (DMSO) for $48 \mathrm{~h}$. Medium containing fresh agonists was added after $24 \mathrm{~h}$. Total RNA was extracted, reverse transcribed and the cDNA was amplified by real-time PCR as described (14) using apoA-II primers (5'-cagacaccaaggacagaga, 5'-cagatggtg aggagtagca) and probe (5'-ctggctaggccgccetccc) (Thermo Electron, Germany). RPLP0 was used as a house-keeping gene; with the primers (5'-agcaagtgggaaggtgtaatcc, 5 '-ccattcta tcatcaacgggtacaa) and probe (5'-tctccacagacaaggccaggac tcgt).

In silico analyses. Approximately $3 \mathrm{~kb}$ of the apoA-II promoter (GenBank accession no. AL590714) was analysed for putative PPREs using the MatInspector program at the Genomatix web site (12). Mulan was used for the sequence comparisons between species (13).

Cloning. The promoter region of apoA-II was cloned by long-range amplification (Roche, USA) according to the manufacturer's instructions using human genomic DNA as template. The primers 5'-tggaacaggaagagtagggaa (-2725 to -2704) and 5'-ctcacacatcttgcctcctta $(+40$ to +61$)$ were used for amplification (Proligo, France). The PCR product was subcloned using the TA-cloning kit (Invitrogen) according to the manufacturer's instructions. A XhoI and KpnI fragment of the apoA-II construct was subsequently transferred to the pGL3-basic vector (Promega, USA). DNA sequencing was performed using Big Dye Terminator Kit and an automatic sequencer (Genetic Analyzer 3100, Applied Biosystems) to confirm that the sequence was in accordance with the genomic DNA sequence.

Transient transfection assays. Plasmids were purified using the Endofree Plasmid Maxi kit (Qiagen, UK). Transfections were performed as described $(11,14)$ and then incubated with GW501516 at different concentrations in DMEM (0.2\% FBS) for $24 \mathrm{~h}$. Subsequently, the cells were harvested and luciferase activity was measured as described (11). Mean values of four replicates for each treatment were calculated.
In vitro mutagenesis. Site-directed mutagenesis of the PPREs was accomplished using a QuickChange Site-directed mutagenesis kit (Stratagene, USA) and mutagenic primers (Proligo, France) corresponding to the $\mathrm{O}$ - and $\mathrm{J}$-site, respectively (Osite: 5'-ggtgacaagtggGGAGGAgAGATTGaagatggggcggg, Jsite: 5'-ggcttctaccAG $\underline{A} G T A a A G \underline{C T T G a a g g c a c c), ~ a c c o r d i n g ~}$ to the manufacturer's instructions. The underlined nucleotides denote mutated sites.

Coupled transcription/translation. The human PPAR $\delta$ sequence was cloned into a TNT vector as described (20). TNT-hPPAR $\alpha$ and TNT-hRXR $\alpha$ were kind gifts from Ingalill Rafter (AstraZeneca, Sweden). In vitro transcription/translation was performed using the rabbit reticulocyte lysate system according to manufacturer's advice (Promega) as described (14).

Electrophoretic mobility shift (EMSA). A double stranded oligonucleotide containing the J-site spanning -740 to -714 of the human apoA-II promoter (J-Wt: 5'-cttctaccAGGGTAaA GGTTGaaggcac) and an identical oligonucleotide containing two point mutations (J-Mt: 5'-cttctaccAG $\underline{A} G T A a A G \underline{C T T G}$ aaggcac) were used for EMSA studies. The rat ACO-PPRE was used as a control (5'-ggaccAGGACAaAGGTCAcgttcgg). EMSA assays were conducted as described (14). In short, $2 \mu 1$ of in vitro produced PPAR $\alpha$ or PPAR $\delta$ together with $2 \mu 1$ of $\mathrm{RXR} \alpha$ or mock lysate (empty vector), was used in each assay. For competition analyses, a 100-fold molar excess of unlabelled double stranded J-Wt, J-Mt or ACO-oligo was added. DNAprotein complexes were run on a $6 \%$ polyacrylamide gel and analysed as described (14).

Chromatin immunoprecipitation (ChIP) assay. HepG2 cells were treated with $100 \mathrm{nM}$ of GW501516 for $8 \mathrm{~h}$ and were subsequently fixed using formaldehyde. Chromatin was purified and sheared using an enzymatic ChIP-IT express kit according to the manufacturer's instructions (Active Motif, USA), followed by immunoprecipitation with a PPAR $\delta$ antibody (sc-7197, Santa Cruz, USA) and an IgG from a ChIP-IT human control kit (Active Motif). Immunoprecipitated DNA was subjected to 36 cycles of PCR with one primer pair amplifying the apoA-II J-site region (apoA-II_J-siteF: 5'-cact gcctagaactgataagg, apoA-II_J-siteR: 5'-ctggatgctgatcactgaaca) and a second pair of control primers amplifying a region $2.5 \mathrm{~kb}$ downstream of the J-site (apoA-II_negF: 5'-tgaatccctcgatttctg gag, apoA-II_negR: 5'-cccggttcaaaagattctcatg) generating a 177 or 253 bp product, respectively. Chromatin input (10\%) was used as positive while $\mathrm{H}_{2} \mathrm{O}$ was used as blank control templates for both primer pairs.

Statistical analyses. In order to establish differences in promoter activity or mRNA expression between control and treated cells, Student's t-tests (two-tailed) were used. Significant differences are indicated as: ${ }^{*} \mathrm{p}<0.05,{ }^{* *} \mathrm{p}<0.01,{ }^{* * *} \mathrm{p}<0.001$.

\section{Results}

GW501516 increases apoA-II gene expression in human hepatoma cells. To analyse whether activation of PPAR $\delta$ regulates apoA-II gene expression in human liver cells, 


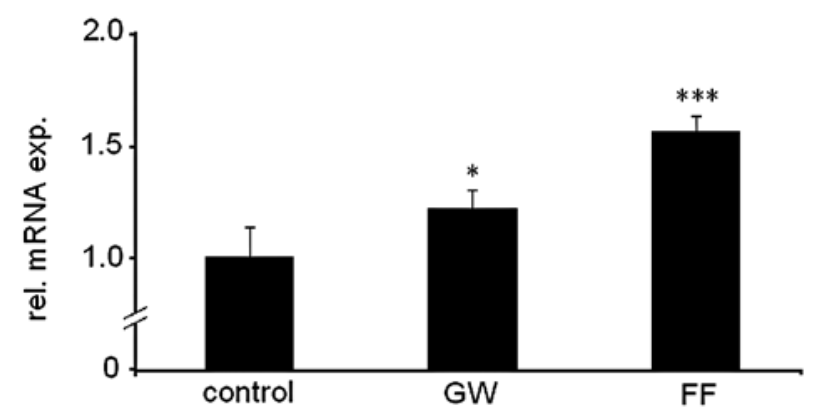

Figure 1. GW501516 (GW) $(100 \mathrm{nM})$ and fenofibric acid $(\mathrm{FF})(500 \mu \mathrm{M})$ induce apoA-II mRNA expression in HepG2 cells after $48 \mathrm{~h}$ of treatment. Results are shown as relative expression compared to cells treated only with vehicle $(\mathrm{n}=4)$. Significant differences: ${ }^{*} \mathrm{p}<0.05,{ }^{* * *} \mathrm{p}<0.001$.

HepG2 cells were treated with GW501516 followed by RNA isolation and cDNA analysis. As shown in Fig. 1, addition of GW501516 (100 nM) under present conditions, increased apoA-II mRNA levels by $1.22 \pm 0.09$ fold, $\mathrm{p}<0.05$. Treatment with $500 \mu \mathrm{M}$ fenofibric acid (FF) was used as a positive control $(1.56 \pm 0.07, \mathrm{p}<0.001)$.

In silico analysis of the apoA-II proximal promoter region reveals two putative PPREs. In order to investigate whether the increase in expression of apoA-II mRNA by PPAR $\delta$ agonist treatment could be due to the presence of PPREs in the promoter region, in silico analyses of the apoA-II promoter region was performed. Two putative PPREs located at -2656 to -2636 and -737 to -717 , respectively, relative to the 5 '-cap site were identified and hereafter designated as the $\mathrm{O}$ - and J-site. Examination of these PPREs revealed that both the O- and J-sites contain 9 out of 13 nucleotides of the core DR1 consensus. Analysis of the 5'-flanking sequence showed that the O-site contains 4 out of 7 nucleotides, while the J-site contains 2 out of 7 nucleotides compared to the consensus sequence (Fig. 2A). In addition, both putative PPREs are conserved in closely related species such as the macaque and chimpanzee. In mice, the DR1 element of the O-site deviates two bases compared with the human sequence showing $92 \%$ homology, whereas the $\mathrm{J}$-site is disrupted by 15 nucleotides (Fig. 2B).

Treatment with PPARS agonist increases apoA-II promoter activity. The proximal promoter of the apoA-II gene encompassing both putative PPREs was cloned into a reporter vector to study whether PPARS induces transcriptional activity. The construct containing $2.7 \mathrm{~kb}$ of the human apoA-II promoter was transiently transfected into two hepatoma cell lines, HepG2 and HuH-7, which were subsequently treated with various doses of GW501516.

In HepG2 cells (Fig. 3A), treatment with $10 \mathrm{nM}$ of GW501516 did not show any significant increase in promoter activity unless a PPAR $\delta$ expression vector was cotransfected, suggesting low endogenous expression of PPAR $\delta$ in these cells $(1.71 \pm 0.93, \mathrm{p}>0.05)$. Accordingly, cotransfection with a PPARd expression vector without any additional treatment increased basal promoter activity up to 6-fold $(6.02 \pm 0.35$, $\mathrm{p}<0.01$ ), whereas treatment with GW501516 in the presence
A

\section{Consensus}

O-site

J-site

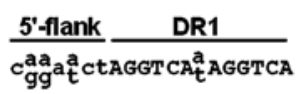

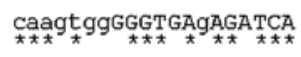

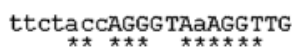

$4 / 7+9 / 13$

$27+9 / 13$

$\begin{array}{cl}\text { B } & \begin{array}{l}\text { Human } \\ \text { Pan Tr. } \\ \text { Macaque } \\ \text { Mouse }\end{array} \\ \text { J-site } & \begin{array}{l}\text { Human } \\ \text { Pan Tr. } \\ \text { Macaque } \\ \text { Mouse }\end{array} \\ & \end{array}$

caagtggGGGTGAgAGATCA caagtggGGGTGAgAGATCA caggtggGGGTGAgAGATCA

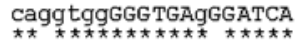

ttctaccAGGGT--

ttctaCCAGGG

ttctaccA.GGT----AAAGGTTG

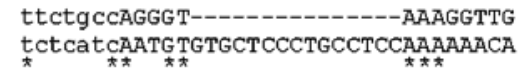

Figure 2. Comparison of putative PPREs. The DR1 nucleotides are given in capital letters while the nucleotides of the 5'-flanking region are shown in lower-case letters. (A) Comparison of identified PPREs in the apoA-II promoter designated as the $\mathrm{O}$-site and $\mathrm{J}$-site, respectively, and the consensus PPRE sequence. The number of matches for the 5'-flanking region and the core DR1, respectively, compared to the consensus sequence are shown to the right. Identical nucleotides between the consensus sequence and the PPREs are denoted by an asterisk. (B) Alignment of genomic sequences in the region of the apoA-II O- and J-sites between human, pan troglodytes (chimpanzee), maqaque and mouse. Identical nucleotides are highlighted by an asterisk.

of PPAR $\delta$ expression increased the promoter activity by 20-fold (20.69 $\pm 0.34, \mathrm{p}=0.001)$. In HuH-7 cells (Fig. 3B), the apoA-II promoter activity increased already at $10 \mathrm{nM}$ GW501516 (1.37 $\pm 0.10, \mathrm{p}<0.05)$. Cotransfection with PPAR alone did not affect the promoter activity significantly $(1.49 \pm 0.26, p>0.05)$, but together with GW501516, the

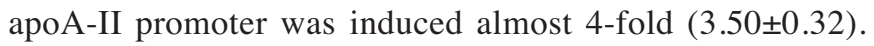
Treatment with $200 \mu \mathrm{M}$ FF or cotransfection with PPAR $\alpha$ alone increased the apoA-II promoter activity in both cell lines (data not shown).

ApoA-II regulation by PPARS is mediated via the J-site. In order to investigate the functional effects of the two putative PPREs in the apoA-II promoter, in vitro mutagenesis of these two sites were performed followed by transfection in $\mathrm{HuH}-7$ cells. The transcriptional activities of the mutated constructs were compared with the activity of the wild-type construct (Fig. 3C). Mutation of both putative PPREs resulted in a loss of induction by PPAR $\delta$. This was also noted for PPAR $\alpha$ (data not shown). However, mutation of the O-site alone did not affect the apoA-II promoter activity after PPAR activation compared to the wild-type construct, whereas mutation of the J-site ablated PPAR $\delta$ and PPAR $\alpha$ activation of the apoA-II promoter. These results indicate that the J-site is the functional PPRE in the promoter region for both PPAR $\delta$ and PPAR $\alpha$. In addition, basal luciferase levels of the constructs containing a mutated J-site (Mt-J, Mt-OJ) were suppressed compared to the activities of the constructs containing either the wild-type (Wt) or the mutated O-site (Mt-O).

PPARS binds to the apoA-II J-site in vitro and in vivo. In order to investigate whether PPAR $\delta$ and PPAR $\alpha$ can bind to 
A

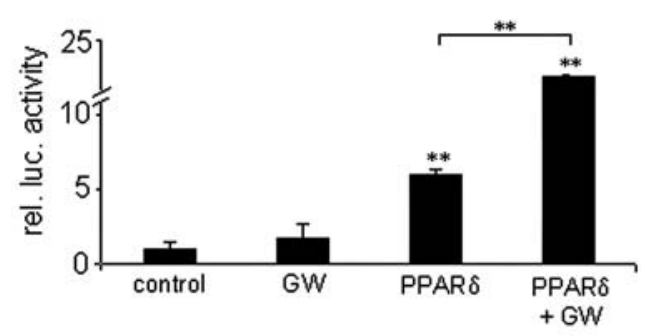

C

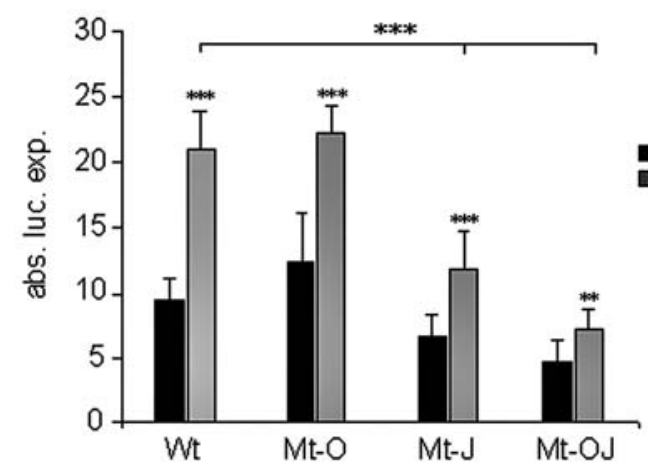

A

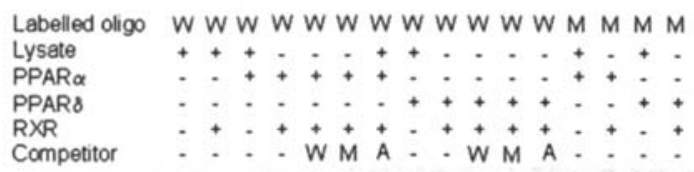

Competitor

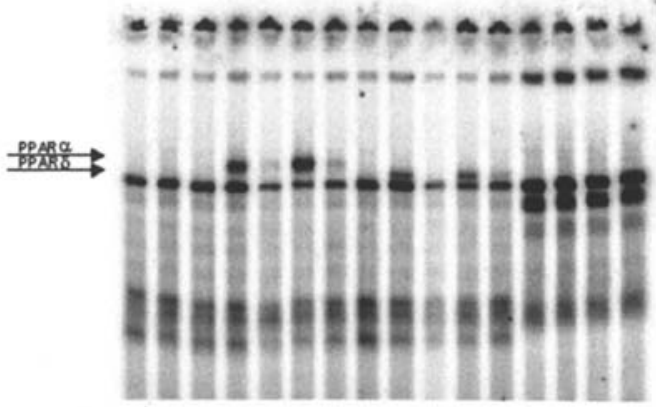

B

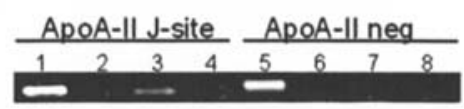

Figure 4. Binding of PPARs to the apoA-II J-site. (A) EMSA showing binding of PPAR $\alpha: \operatorname{RXR} \alpha$ and PPAR $\delta: \operatorname{RXR} \alpha$ heterodimers to the wild-type (W) but not to the mutated (M) J-site. Labelled wild-type or mutated J-site specific oligonucleotides were incubated with in vitro synthesized human PPARs and RXR $\alpha$. Competition assay was performed by adding 100-fold excess of cold wild-type (W), mutant (M) or ACO-PPRE (A) probes. Arrows indicate the positions of the PPAR $\alpha$ RXR and PPAR $\delta: R X R$ DNA:protein complexes, respectively. (B) HepG2 cells were treated with GW501516 (100 nM) for $8 \mathrm{~h}$, fixed and chromatin was purified followed by enzymatic digestion. ChIPs were performed using a PPARS antibody and negative control IgG. Immunoprecipitated DNA was subjected to PCR with a primer pair specific to the apoA-II J-site (lanes 1-4). As a negative control a second set of primers was used to amplify a region $2.5 \mathrm{~kb}$ downstream of the J-site (apoA-II neg, lanes 5-8). PCR was performed on DNA isolated through ChIP using chromatin input as a positive control (lanes 1 and 5), negative control IgG (lanes 2 and 6), PPAR $\delta$ antibody (lanes 3 and 7) and blank $\mathrm{H}_{2} \mathrm{O}$ (lanes 4 and 8).

B

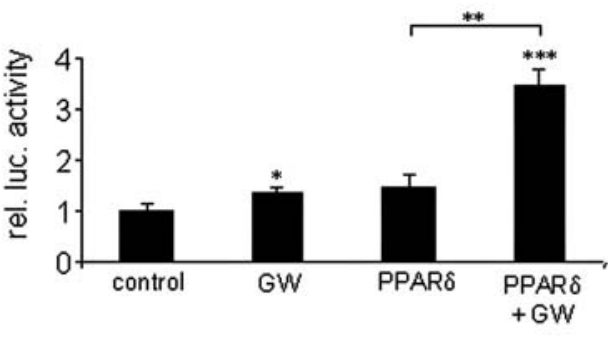

Figure 3. Effects of GW501516 and/or PPARd overexpression on apoA-II promoter activity in (A) HepG2 and (B) HuH-7 cells. The luciferase activity of transfected untreated control cells in each experiment was set to one and the resulting activities for the test agents were calculated relatively to the control cells $(n=4)$. (C) Transfection assays in HuH-7 cells using constructs with mutated PPREs in the presence or absence of PPAR $\delta$ overexpression together with GW501516 treatment $(10 \mathrm{nM})$. Black bars show promoter activity in cells transfected with the wild-type (Wt) or mutated (Mt-O, Mt-J or Mt-OJ) constructs in the absence of PPARS overexpression or GW501516. Grey bars denote luciferase activity in cells transfected with wild-type or mutated constructs and PPAR $\delta$ expression plasmid, which were subsequently treated with GW501516 $(10 \mathrm{nM})$ for $24 \mathrm{~h}(\mathrm{n}=4)$. Significant differences compared to untreated controls: ${ }^{*} \mathrm{p}<0.05,{ }^{* *} \mathrm{p}<0.01,{ }^{* * *} \mathrm{p}<0.001$.

the J-site located at -737 to -717 in the apoA-II promoter, electrophoretic mobility shift assays (EMSA) were performed. Human PPAR $\delta / R X R \alpha$ as well as PPAR $\alpha / R X R \alpha$ heterodimers resulted in the formation of retarded complexes, which were not detected using RXR $\alpha$, PPAR $\delta$ or PPAR $\alpha$ alone (Fig. 4A). No binding was observed when the J-site was mutated. Competition studies using unlabelled apoA-II_J-PPRE or ACO-PPRE sequences diminished protein:DNA complex formation, whereas no change was seen when a mutated apoA-II_J-probe was used. To confirm that PPARd binds to the J-site also in vivo, a chromatin immunoprecipitation (ChIP) assay was performed. Immunoprecipitated chromatin DNA using a PPARS antibody, generated a PCR-product with a primer pair flanking the J-site in the apoA-II promoter (Fig. 4B, lane 3) but not with a pair of negative control primers binding $2.5 \mathrm{~kb}$ downstream (Fig. 4B, lane 7). ChiP analysis using a negative control antibody did not give rise to any PCR-product with neither of the primer pairs (Fig. 4B, lanes 2 and 6). Thus, the ChIP assay confirmed binding of PPAR $\delta$ to the J-site.

\section{Discussion}

PPARS has received a great deal of attention during recent years since PPAR $\delta$ activation has been shown to elevate plasma HDL and decrease triglyceride levels in primates. We previously performed a microarray cDNA analysis comparing HepG2 cells treated with the PPAR $\delta$ specific ligand, GW501516, with untreated cells in order to identify PPARS target genes involved in lipid metabolism. The data from this screening indicated that GW501516 treatment results in increased expression of apoA-II (unpublished data). 
Plasma apoA-II levels are controlled mainly by synthesis in the liver rather than catabolism (15) and is one of the major apolipoproteins of HDL recently assigned to possess beneficial properties in HDL cholesterol metabolism and atherosclerosis (3).

This study investigated the effects of PPAR $\delta$ on apoA-II gene expression and was designed to identify functional PPREs involved in the transcriptional regulation. Although, the observed induction of the apoA-II gene expression by PPAR $\delta$ activation in this study seems modest, the regulation of the apolipoprotein genes in vitro has been shown to be highly dependent on the batch of serum used. Induction of apoA-II mRNA expression by the same concentration of fibrates has been reported to vary from 1.5- to 2.5-fold using different batches of serum, both within and between studies $(7,16)$. In the present study, the mRNA expression of apoA-II increased by 1.5 -fold after treatment with fibrates, indicating that the batch of serum used may contribute to the overall moderate effect on apoA-II mRNA expression. Thus, the magnitude of induction might not be as important as the reproducibility of the activation itself.

The results from reporter gene assays in this study, where PPAR $\delta$ activation resulted in a 4-fold increase in promoter activity using $\mathrm{HuH}-7$ cells and as much as a 20 -fold increase using HepG 2 cells, are further evidence of a PPAR $\delta$ inducing effect on the apoA-II promoter in vitro. Accordingly, obese rhesus monkeys treated with GW501516 show plasma apoA-II elevations of approximately $21 \%$, indicating that apoA-II expression is induced by PPAR $\delta$ activation also in vivo (8).

The results of this study show that the effect of PPAR $\delta$ activation on the human apoA-II promoter is mediated via a PPRE located at -737 to -717 , upstream of the cap site, which corresponds to the regulatory J-site as determined by DNase I footprinting analyses (6). The putative PPRE in the apoA-II promoter positioned at -2656 to -2636 relative to the cap site, the O-site, has not been analysed before. Although, this site is conserved between several species and shows a high degree of homology to the PPRE consensus sequence, it does not seem to play a role in the transcriptional regulation of the human apoA-II gene by PPARs. One of the reasons might be that this site is located within an intron of the TOMM40L gene. The apoA-II and TOMM40L genes are organised in a head-to-head fashion and only 2414 bp separates the transcriptional start sites of the respective genes. The function of TOMM40L is as yet unknown but studies have shown that this gene is transcribed (17).

The J-site is the same functional PPRE that has been shown to mediate the effects of PPAR $\alpha$ activation (7), which is not surprising since all the PPARs are reported to share a common consensus binding site on the DNA. However, it has been reported that the 5'-flanking region of the PPRE is of importance for PPAR isotype binding of DNA and that some PPARs can bind to imperfect PPRE sequences whereas others cannot, intriguingly suggesting distinct roles for different PPAR family members (5). The J-site constitutes an important regulatory region in the apoA-II promoter, as these data suggest, since the basal transcriptional activity decreased when the site was mutated, which is in agreement with a previous study (7). Of note, the J-site has been shown to be important not only for mediating the effects of PPARs but also for binding of hepatocyte nuclear factor- $4 \alpha$ and other members of the nuclear receptor family (18). In addition, others have shown that the region located -911 to -614 encompassing the $\mathrm{J}$-site is required for basal transcription from the apoA-II promoter (6).

In conclusion, we have shown that the human apoA-II gene is transactivated by PPAR $\delta$ through binding to the J-site in the promoter. Since increased synthesis of apoA-II is correlated with elevated plasma HDL cholesterol concentrations, apoA-II might be one of many genes responsible for the beneficial effects regarding lipid and lipoprotein metabolism observed as a result of PPAR $\delta$ activation.

\section{Acknowledgements}

This study was supported by the Swedish Medical Research Council (12659), the Swedish Heart-Lung Foundation, the Swedish Diabetes Foundation, Professor Nanna Svartz Foundation, Magn. Bergvalls Foundation, the Foundation for Old Servants and the Karolinska Institutet. P.T. is supported by a stipend from the Swedish Heart-Lung Foundation.

\section{References}

1. Nofer JR, Kehrel B, Fobker M, Levkau B, Assmann G and von Eckardstein A: HDL and arteriosclerosis: beyond reverse cholesterol transport. Atherosclerosis 161: 1-16, 2002.

2. Tailleux A, Duriez P, Fruchart JC and Clavey V: Apolipoprotein A-II, HDL metabolism and atherosclerosis. Atherosclerosis 164: $1-13,2002$.

3. Birjmohun RS, Dallinga-Thie GM, Kuivenhoven JA, Stroes ES, Otvos JD, Wareham NJ, Luben R, Kastelein JJ, Khaw KT and Boekholdt SM: Apolipoprotein A-II is inversely associated with risk of future coronary artery disease. Circulation 116: 2029-2035, 2007.

4. Barbier O, Torra IP, Duguay Y, Blanquart C, Fruchart JC, Glineur C and Staels B: Pleiotropic actions of peroxisome proliferator-activated receptors in lipid metabolism and atherosclerosis. Arterioscler Thromb Vasc Biol 22: 717-726, 2002.

5. Juge-Aubry C, Pernin A, Favez T, Burger AG, Wahli W, Meier CA and Desvergne B: DNA binding properties of peroxisome proliferator-activated receptor subtypes on various natural peroxisome proliferator response elements. Importance of the 5'-flanking region. J Biol Chem 272: 25252-25259, 1997.

6. Chambaz J, Cardot P, Pastier D, Zannis V and Cladaras C: Promoter elements and factors required for hepatic transcription of the human ApoA-II gene. J Biol Chem 266: 11676-11685, 1991.

7. Vu-Dac N, Schoonjans K, Kosykh V, Dallongeville J, Fruchart JC, Staels B and Auwerx J: Fibrates increase human apolipoprotein A-II expression through activation of the peroxisome proliferator-activated receptor. J Clin Invest 96: 741-750, 1995.

8. Oliver WR Jr, Shenk JL, Snaith MR, Russell CS, Plunket KD, Bodkin NL, Lewis MC, Winegar DA, Sznaidman ML, Lambert MH, Xu HE, Sternbach DD, Kliewer SA, Hansen BC and Willson TM: A selective peroxisome proliferator-activated receptor delta agonist promotes reverse cholesterol transport. Proc Natl Acad Sci USA 98: 5306-5311, 2001.

9. Sprecher DL, Massien C, Pearce G, Billin AN, Perlstein I, Willson TM, Hassall DG, Ancellin N, Patterson SD, Lobe DC and Johnson TG: Triglyceride: high-density lipoprotein cholesterol effects in healthy subjects administered a peroxisome proliferator activated receptor delta agonist. Arterioscler Thromb Vasc Biol 27: 359-365, 2007.

10. Barish GD, Narkar VA and Evans RM: PPAR delta: a dagger in the heart of the metabolic syndrome. J Clin Invest 116: 590-597, 2006.

11. Glinghammar B, Skogsberg J, Hamsten A and Ehrenborg E: PPARdelta activation induces COX-2 gene expression and cell proliferation in human hepatocellular carcinoma cells. Biochem Biophys Res Commun 308: 361-368, 2003. 
12. Cartharius K, Frech K, Grote K, Klocke B, Haltmeier M, Klingenhoff A, Frisch M, Bayerlein M and Werner T: MatInspector and beyond: promoter analysis based on transcription factor binding sites. Bioinformatics 21: 2933-2942, 2005.

13. Ovcharenko I, Loots GG, Giardine BM, Hou M, Ma J, Hardison RC, Stubbs L and Miller W: Mulan: Multiple-sequence local alignment and visualization for studying function and evolution. Genome Res 15: 184-194, 2005.

14. Lundell K, Thulin P, Hamsten A and Ehrenborg E: Alternative splicing of human peroxisome proliferator-activated receptor delta (PPAR delta): effects on translation efficiency and transactivation ability. BMC Mol Biol 8: 70, 2007.

15. Ikewaki K, Zech LA, Kindt M, Brewer HB and Rader DJ Jr: Apolipoprotein A-II production rate is a major factor regulating the distribution of apolipoprotein A-I among HDL subclasses LpA-I and LpA-I:A-II in normolipidemic humans. Arterioscler Thromb Vasc Biol 15: 306-312, 1995.
16. Clavey V, Copin C, Mariotte MC, Bauge E, Chinetti G, Fruchart J, Fruchart JC, Dallongeville J and Staels B: Cell culture conditions determine apolipoprotein CIII secretion and regulation by fibrates in human hepatoma HepG2 cells. Cell Physiol Biochem 9: 139-149, 1999.

17. Simpson JC, Wellenreuther R, Poustka A, Pepperkok R and Wiemann S: Systematic subcellular localization of novel proteins identified by large-scale cDNA sequencing. EMBO Rep 1: 287-292, 2000.

18. Ladias JA, Hadzopoulou-Cladaras M, Kardassis D, Cardot P, Cheng J, Zannis V and Cladaras C: Transcriptional regulation of human apolipoprotein genes ApoB, ApoCIII, and ApoAII by members of the steroid hormone receptor superfamily HNF-4, ARP-1, EAR-2, and EAR-3. J Biol Chem 267: 15849-15860, 1992. 